\title{
Research progress of preservative coating of natural polymer materials
}

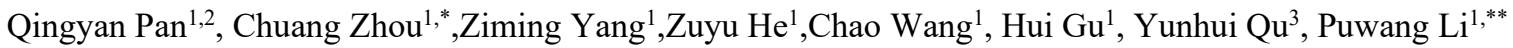 \\ ( ${ }^{1}$ South Subtropical Crop Research Institute of Chinese Academy of Tropical Agricultural Sciences, Key Laboratory of Hainan \\ Province for Postharvest Physiology and Technology of Tropical Horticultural Products, Zhanjiang 524091,P.R.China; \\ 2. College of Food Science and Technology, Huazhong Agricultural University, Wuhan 430070,P.R.China; \\ 3. Agricultural Product Processing Research Institute of Yunan Academy of Agricultural Sciences, Kunming, Yunnan 650000, \\ P.R.China)
}

\begin{abstract}
The planting area and output of fruits and vegetables in China are the first in the world. However, due to the imperfection of storage and transportation fresh-keeping technology, the annual loss of fruits and vegetables in China is serious. Rational use of fresh-keeping coating is of great significance for improving the shelf life of fruits and vegetables. Natural polymer preservative coating is widely concerned in the field of fruits and vegetables due to its wide sources, degradability, low price and good film-forming ability. In this paper, the types of natural polymer preservative coating were reviewed, and its application in the freshkeeping of fruits and vegetables was summarized and prospected, so as to provide reference for further research.
\end{abstract}

\section{Introduction}

With the improvement of living standards and the environmental protection consciousness, people also gradually increase to the requirement of the technology for fruits and vegetables, although many technology developed at home and abroad, but each technology has its drawbacks, especially chemical preservatives residual damage is becoming more and more attention to human health, so researchers tend to gradually develop security, non-toxic, highly effective, type of preservatives added. Preservatives are mainly divided into natural biological preservatives and chemical preservatives. Chemical preservatives have relatively good anti-corrosion effect and are convenient to use, but they have certain safety risks and may cause chemical pollution. As a natural biological preservative, natural polymer preservative coating has advantages of safety, high efficiency, low cost, suitable for small-batch processing, and suitable for large-batch preservation, etc., so it has become a research hotspot in recent years [1]. The fresh fruits and vegetables coated with film can not only maintain good quality in the storage period, but also extend the shelf life, so people pay more and more attention to it.

\section{The types of natural polymer preservative film}

At present, the main methods of fresh-keeping and storage of fruits and vegetables include air-conditioned fresh-keeping and low-temperature refrigeration. However, due to its large storage scale, high equipment requirements and poor preservation effect, it is difficult to meet the demand of large-scale and low-cost circulation. In recent years, the preservation method of coating film is developing rapidly and has achieved good results. Natural polymer with sources, biodegradable, price is low wait for a characteristic, preparation of natural polymer modified into fresh keeping film, awaiting the became a very uniform layer of film after drying, can effectively isolate the fruit with air for gas exchange, which can inhibit the respiration of the fruit, reduce the consumption of nutrients, is a good way of keeping fresh [2]. Natural macromolecule refers to macromolecule that exists in nature, such as polysaccharide macromolecule such as chitosan, starch and cellulose, and protein macromolecule such as soybean protein, wheat protein and corn protein, as well as lipid macromolecule such as microbial co-polyester and natural wax. All of these natural polymers can be used to prepare fresh-keeping coatings to enhance the fresh-keeping of fruits and vegetables. According to the different substrate materials, the preservative coating film of natural macromolecule can be divided into three types, namely polysaccharide (chitosan, starch, cellulose, etc.), protein (corn protein, wheat protein, soybean protein, etc.), and lipid (microbial co-polyester, natural wax, etc.).

\section{Polysaccharide preservative coating and its application}

The coating materials of natural high molecular polysaccharide mainly include starch, cellulose, chitosan

\footnotetext{
* Corresponding author:zhouchuang0759@163.com

**puwangli@163.com
} 
and so on. These coatings usually have good barrier property to gas and can form uniform film on the surface of fruits and vegetables

\subsection{Chitosan preservative coating}

Chitosan is a chitin deacetylated derivative with excellent properties such as natural, safety, biocompatibility and non-toxicity. In recent years, chitosan, as an excellent membrane material, has great advantages in food preservation and consumption, attracting increasing attention [3]. Chitosan has good film-forming performance, and a film can be formed on the surface of fruits and vegetables by coating or soaking, thus forming a micro-air-regulating environment, which can not only prevent the fruit from losing water, but also effectively prevent the invasion of microorganisms, reduce the fruit rot to a certain extent, and thus extend the storage time of the fruit. In recent years, with the increasing demand for healthy food and the increasing demand for safe, non-toxic and degradable materials, it is an urgent problem to study the structure and filmforming properties of chitosan in a deep and systematic way in the field of fresh-keeping. The results show that chitosan coating can be divided into single coating of chitosan and composite coating of chitosan.

\subsubsection{Single coating of chitosan}

The coating agent mainly refers to the material that can cover the surface of food for quality preservation, freshness preservation and water evaporation. Chitosan's good film-forming and antibacterial properties make it show a broad application prospect in the field of fruit and vegetable coating and fresh-keeping packaging materials. The results of previous studies showed that both concentration and molecular weight had effects on the preservation of chitosan. Zhao Yumei [4] studied the effects of chitosan solution coating treatment with different concentrations on post-harvest storage quality of grape. It was found that the activity of polyphenol oxidase and peroxidase in grapes treated with $2 \%$ chitosan solution remained at a low level at the 13th day after harvest, which effectively delayed the aging process of grapes. Zhang et al. [5] treated with chitosan with different molecular weights on the aging of nectarine fruits stored at $25^{\circ} \mathrm{C}$ for 8 days after harvest. It was found that the treatment of chitosan with low molecular weight and high polymer could delay the aging of fruit and enhance its antioxidant capacity by inhibiting respiration rate. At the same time, fruits treated with high molecular weight chitosan had better quality and REDOX status than those treated with low molecular weight chitosan. Kittur et al. [6] applied chitosan to preserve bananas and mangoes by coating at $27^{\circ} \mathrm{C}$ and $65 \%$ of relative humidity. The test results showed that coating treatment could reduce weight loss of the two fruits, effectively inhibit microbial infection, and the storage effect was better than carboxymethyl cellulose membrane. Ghaouth et al. [7] used chitosan coating to keep fresh strawberries. The test results showed that at $13^{\circ} \mathrm{C}$ and after 29 days of storage, the rot rate of chitosan coating strawberry was $20 \%$, while that of uncoated strawberry was as high as $82 \%$. Jiang et al. [8] investigated the preservation effect of chitosan coating on strawberry. The results showed that chitosan coating could effectively improve the overall quality of strawberry and prolong the shelf life of strawberry by 2 days, which had great potential in the industrial application of fruit preservation.

Chitosan as a new type of fresh material, because of its good film forming and antibacterial favored by researchers, but the poor solubility of chitosan, single chitosan membrane exists high permeable rate and easy rupture of membrane, the shortcomings of easy broken, can't satisfy the preservation of fruits and vegetables storage, so will be in the process of practical application of chitosan with other antibacterial material compound after use, this not only can make up for its shortcomings when used alone, also can strengthen film inhibitory activity of some pathogenic microorganisms [9]. When choosing composite materials, it is necessary to consider their toxicity and whether they can be degraded, so as to ensure their safety and environmental friendliness during use.

\subsubsection{Chitosan composite film}

Chitosan composite film formation refers to the improvement of chitosan thin film with film forming agent, natural preservative, antibacterial group and other auxiliary agents, so that chitosan thin film has better physical and chemical properties such as film-forming property, permeability, antibacterial property, mechanical property and antioxidant property. Torabi et al. [10] prepared acrylic resin with butyl acrylate and glycidyl methacrylate as raw materials by evaporative casting method, and then compounded with chitosan to prepare antibacterial composite film. Its antibacterial properties were investigated by toxicological tests of The bacteria and Escherichia coli. The results showed that the antibacterial effect of high content chitosan composite film was greater than that of low content chitosan composite film. Siripatrawan et al. [11] mixed chitosan with propolis extract to prepare composite membrane. The results showed that propolis/chitosan composite film had a good inhibitory effect on Staphylococcus aureus and Salmonella, and the composite film could be well used in food active packaging materials.

In the past studies, some researchers have improved the solubility and antibacterial properties of chitosan by constructing organic acid and chitosan composite materials, which has been developed and utilized in the aspect of membrane preservation. Shi et al. [13] studied the influence of graft copolymer of chitosan (CTS) and salicylic acid (SA) (CTS-G-SA) on the storage resistance of grapefruit fruit after harvest. It was found that after graft copolymer treatment, the green mold caused by penicillium fingertip could be significantly inhibited, and the fruit hardness could be maintained without damaging the fruit quality. In addition, graft copolymer treatment 
can also inhibit the dissolution of cell wall polysaccharides by inhibiting the activity and gene expression of polygalacturonase, cellulase, pectinmethyl esterase and other enzymes, thus inhibiting the fruit rot and delaying the fruit softening.

In recent years, essential oils have attracted more and more researchers' interest because of their non-toxicity and high selectivity [14]. Essential oil can not only effectively improve the anti-corrosion and antibacterial effect of the composite film, but also improve the mechanical properties of the composite film, thus making the fresh-keeping effect more obvious [15]. Hafsa et al. [16] prepared chitosan composite film containing eucalyptus globulus essential oil by solution casting and solvent evaporation. The results showed that the compound film with eucalyptus globulus essential oil had some antibacterial properties and significant inhibitory activity against Staphylococcus aureus. Mohammadi et al. [17] reported that the combination of chitosan (CS) and ZEO (ZEO) or Cinnamomum occidentalis (CEO) essential oil on cucumber in vitro and artificially infected cucumber could inhibit the pathogenic bacteria (Phytophthora Diogenes) that led to the decomposition of cucumber fruit, and the cucumber treated with combination ( $\mathrm{CC}$ and $\mathrm{CZ}$ ) could reduce the fungal decay to the range of $77-85 \%$ on the 9 th day.

Finally, the composite application of chitosan with other antibacterial materials can not only make up for its disadvantages when used alone, but also enhance the inhibitory activity of the film against some pathogenic microorganisms. But at present, there are still many problems in the application of composite membrane. Part antibacterial material is no synergistic effect with chitosan, the composite membrane effect is only several material effect of linear superposition, therefore, should focus on the future research direction of antimicrobial materials and the study of fit between chitosan, and according to the mechanism of selecting suitable fruit varieties, and then solve the problems of fruit postharvest losses in agricultural production process.

\subsection{Starch preservation coating}

Starch, as a kind of renewable natural polymer material, has been increasingly used to make antibacterial packaging film. Antibacterial starch film is based on starch and made of other antibacterial materials. It has the advantages of safety, health, good performance, low price, green and degradable. According to the different chemical composition of antibacterial materials, antibacterial starch film can be divided into inorganic, synthetic and natural antibacterial starch film.

\subsubsection{Inorganic antibacterial agent starch film}

The first is metal ions as antibacterial substances, silver ions are the most widely used. In the aqueous solution of starch, Duan et al. [18] reduced silver nitrate into nano silver particles, and poured the solution into the glass dish to make the starch film containing nano silver particles. The results showed that the release of the oxidized negative particles in the liquid environment made the starch membrane very resistant to Staphylococcus aureus. After $1 \mathrm{~h}$ of exposure, the growth of microorganisms was detected to decrease by about $99 \%$. The second is the use of photochemical reactions of atomic oxygen to sterilize (such as zinc oxide and titanium dioxide). Sunil et al. [19] prepared the starch film containing zinc oxide by in-situ generation method, and studied its hygroscopicity and antimicrobial properties. The results showed that the starch film containing zinc oxide not only showed clear bacteriostatic circle to Escherichia coli, but also had much lower moisture absorption than the single starch film. The results showed that the starch film containing zinc oxide could be used to inhibit the growth of bacteria on food surface. But this kind of antibacterial agent must oxygen, the presence of water or ultraviolet radiation to play a bactericidal effect.

\subsubsection{Synthetic antiseptic starch film}

Synthetic antibacterial agent refers to a class of antibacterial compounds made by artificial synthesis, adding to starch film can also improve the antibacterial property of starch film. Common synthetic antimicrobials include alcohols, imidazoles, organic acids, organic salts, etc. Sorbic acid and its salts are widely used in food packaging because of their good bacteriostatic effect.

Silvia et al. [20] prepared cassava starch containing potassium sorbate antibacterial film under different gelatinization/drying conditions using cassava starch, glycerin, potassium sorbate and water as raw materials. The results showed that the antibacterial starch film could not only prevent the contamination of Bayerbound yeast, but also inhibit the growth of yeast in semisolid products with high acidity and high water activity. Bilal et al. [21] added ascorbic acid to rice starch and glycerin to make antibacterial film by the method of drainage. The results showed that the antibacterial film of rice starch containing ascorbic acid had better antibacterial property in the simulated aqueous food solution.

The results show that synthetic antibacterial agent can kill bacteria faster than inorganic antibacterial agent when used in food packaging. But the heat resistance and poor stability of synthetic antimicrobials limit their application. Therefore, the content of starch must be strictly controlled within the scope of regulations when it is added to starch film.

\subsubsection{Starch film of natural antibacterial agent}

Natural antimicrobials are extracted from animals, plants and natural minerals. They are safe and hygienic, harmless to human body and have good antimicrobial properties. Natural antibacterial agents mainly include chitosan, plant extracts (such as essential oils, lignin, etc.) [22-24], antibiotics (such as natamycin, streptococcus lactis peptide, etc.), etc., which can be combined with starch to make antibacterial starch film. Zhong Yu et al. 
[25] studied the preparation technology of -chitosan and pueraria starch composite membrane and its antibacterial properties against Escherichia coli. The results showed that when the mass ratio of -chitosan to pueraria starch was $1: 1$, the prepared composite membrane reduced the number of E. coli by $8.3 \mathrm{CFU} / \mathrm{m} \mathrm{L}$ within $24 \mathrm{~h}$. The essential oil in plant extract contains high concentration of phenolic compounds, which has good antibacterial effect. Mehran et al. [26] added wild rose and peppermint essential oils to the cornstarch membrane and studied their antibacterial properties against Staphylococcus aureus. The two kinds of films showed better antibacterial property, among which the corn starch film containing wild rose oil had better antibacterial property against Staphylococcus aureus than the corn starch film containing peppermint oil. It is proved that both wild rose oil and peppermint oil have the potential to be added directly into corn starch to prepare degradable antibacterial film for food packaging. Carolina et al. [27] also studied the effect of starch membranes containing natamycin and Streptococcus lactis on the microbial stability of frozen Argentine cheese. It was found that the starch film containing Streptococcus lactis inhibited the growth of yeast and mold at cold storage temperature. Therefore, the antibacterial starch film containing Streptococcus lactis is a very effective method to prevent cheese contamination and improve the stability of surface microorganisms.

\subsection{Cellulose preservative coating}

Cellulose is the most abundant and most widely distributed polysaccharide in nature. It is a large molecule polysaccharide composed of glucose, insoluble in water and general organic solvents, and is the main component of plant cell wall. The preservative coating made of cellulose has good film-forming property, but the permeability barrier of gas is not good, glycerin and protein are usually needed to improve the performance. According to the different chemical composition of composite materials in cellulose preservation film, cellulose preservation film can be divided into cellulose/synthetic polymer preservation film and cellulose/natural polymer preservation film.

\subsubsection{Cellulose/synthetic polymer preservative coating}

Roohanidd et al. [28] added cellulose to the polyvinyl alcohol with a film agent to form a composite film. The test results showed that there was a good synergy between the two, and the tensile strength and elastic modulus of the composite film increased significantly with the increase of cellulose content. Ljungberg et al. [29] added cellulose to the solubilized polypropylene to form a composite film, and the test results showed that cellulose could be uniformly dispersed into the polypropylene matrix, and the mechanical properties of the polypropylene film were improved. Aranguren et al. [30] added cellulose to polyurethane to form a composite film. The test results showed that there was a strong interaction between the two and the mechanical properties of the composite film were significantly improved. Therefore, the mechanical properties of cellulose and synthetic polymer coating can be obviously improved, and it has great potential in the industrial application of fruit preservation.of its good natural properties. In vitro drug release experiments found that loaded DOX superparamagnetic Fe3O4 sodium alginate nanospheres prepared by coprecipitation were much faster in acidic environments $(\mathrm{pH}$ 5.0) than in alkaline environments ( $\mathrm{pH}$ 7.4) [24]. The study found that magnetic alginate microspheres are easier to control and target release anticancer drugs, reducing side effects and achieving higher results.

\subsubsection{Cellulose/natural polymer preservative coating}

At present, the cellulose and natural polymer composite coating has been widely used, Dogan, etc. [31] will be added to the hydroxypropyl methyl cellulose composite membrane, which was formed in the test results show that the water vapor transmittance composite membrane is reduced, mechanical performance is good, is conducive to broaden the hydroxypropyl methyl cellulose as application field of food packaging materials and value. Alemdar[32] prepared the composite film from cellulose and starch, and the test results showed that cellulose could be uniformly dispersed into the starch matrix, the tensile strength and elastic modulus of the composite were significantly increased, and the glass transition temperature was also significantly increased. Petersson et al. [33] added modified cellulose to polylactic acid matrix to form a composite film. The test results showed that cellulose could be well dispersed into polylactic acid, and the thermal stability and energy storage modulus of the composite film were greatly improved. Therefore, the properties of the composite film can be improved by the combination of cellulose and natural polymer.

\section{Protein preservative coating and its applicationand Outlook}

Protein film has the characteristics of wide source, good taste and high nutritional value, and its preparation into fresh coating film can improve the quality of fruits and vegetables and processed products. Zein film, wheat gluten film and soybean protein isolate film are the main protein preservative films. The results show that the protein cling film is an ideal material with good air resistance and little permeability, which not only has good fresh-keeping function, but also has certain nutritional value.5 Acknowledgements

\subsection{Zein membrane}

Zein mainly comes from corn and has the characteristics of easy film formation, oxygen resistance and good water resistance. However, pure zein film is fragile, so 
some plasticizers and cross-linking agents can be added or the process conditions can be optimized to improve the performance of zein film. The coating film has good moisture resistance and gas resistance, and can be used to keep fresh fruits and vegetables. Ouyang Xiaoyan et al. [34] studied the application of zein film formation in citrus preservation, and showed that $0.5 \%$ glycerol was used as plasticizer, $90 \%$ acetic acid was added to extract zein, and the obtained zein film was applied to the surface of citrus to obtain the best preservation effect. Li guijuan et al. [35] extracted zein from zein powder and decolorized zein to prepare zein film. The results showed that the film prepared by the extracted zein had better preservation effect and could prolong the storage period of cherry tomatoes.

\subsection{Wheat gluten protein membrane}

Gluten, commonly known as gluten, is a kind of natural protein, mainly composed of glutenin and gliadin. Among them, glutenin is more elastic and gliadin has better elongation. Therefore, wheat gluten protein can be made into plastic wrap, which has a broad application prospect. Chen Xinjian et al. [36] carried out litchi preservation experiment under normal temperature by wheat gluten protein coating treatment. According to the changes of skin Browning, sensory index, rotten degree and nutrient composition during storage, the freshkeeping period of litchi could be extended from 3 days to 7 days by this kind of fresh-keeping technology. Tong Ying et al. [37] showed that the modified gluten coating treatment could significantly prolong the storage period of soft jujube kiwifruit, with good sensory quality, and significantly improve the moisture content, VC content and hardness.

\subsection{Soybean protein isolate membrane}

Among all kinds of biopolymer materials, soybean protein isolate (SPI) has attracted much attention due to its wide sources, environmental friendliness, low price, natural and renewable properties. Although the advantages of SOYBEAN protein isolate (SPI) are outstanding, the membrane made of pure SOYBEAN protein isolate (SPI) still has many disadvantages. In order to improve the comprehensive properties of THE membrane, modification method is usually used to treat the membrane. Martell et al. [38] found that the tensile strength of the spI film increased from $(6.1 \pm 0.6) \mathrm{MPa}$ to 8.4 $\mathrm{MPa}$ after adding the mechanically treated nanocellulose with a mass fraction of $5 \%$. The nanofibers were treated with 5\% (mass fraction) enzyme. The tensile strength of soybean protein isolate film increased from $(6.1 \pm 0.6) \mathrm{MPa}$ to $9.0 \mathrm{MPa}$. It should be noted that the addition of mechanically treated nanocellulose with a mass fraction of $5 \%$ significantly reduced the flexibility of the MEMBRANE, while the addition of $5 \%$ enzyme-treated nanocellulose had much less effect on the flexibility of the membrane. Han et al. [39] found that the tensile strength of soybean isolate films was significantly improved after adding an appropriate amount of licorice residue extract. This may be due to the interaction between components of licorice extract (e.g., flavonoids) and soy protein isolate (SPI) membranes resulting in strong interfacial adhesion, which helps to resist fracture more effectively.

Soybean protein isolate (SPI) membrane has been widely studied because of its excellent properties after modification. At present, the application of preservation is the most extensive research direction of soybean protein isolate membrane. Wu et al. [40] prepared film solution with $10 \%$ acylated soy protein isolate, $0.34 \%$ chitosan with mass fraction and $0.2 \%$ stearic acid as raw materials and coated apples for $60 \mathrm{~s}$, the peak of respiratory jump of apples could be delayed until the $3 \mathrm{rd}$ week. Liu et al. [41] coated apples with a film solution made by mixing large isolated protein beans with a mass fraction of $10 \%$ and nano-Siox with a mass fraction of $0.3 \%$ for $60 \mathrm{~s}$, and the peak of respiratory jump of apples could be delayed until the 5 th week.

To sum up, although the membrane made of soybean protein isolate has great application prospect, many adverse factors limit its large-scale application, and its large-scale commercial application still needs a long way to go.

\section{Lipid preservative coating and its application}

The lipid coating materials used for fruits and vegetables mainly include waxy and paraffin film coatings and resin based film coatings. Zamudio et al. [42] combined candlestick wax and Bacillus subtilis strains to preserve strawberries with coating film, and found that this coating film could reduce the fruit rot rate on the third day, and had an inhibitory effect on rhizopus fungi, thus extending the shelf life of strawberries. Hiumarelli et al. [43] coated fresh-cut apples with carnauba wax and cassava starch combined to improve water resistance. Mehyar et al. [44] compound walnut coating with whey protein isolate and Carnauba carnauba wax $(1: 1: 1)$ can significantly improve the fresh and taste of walnut, but if the ratio is adjusted to $1: 1: 2$, it will cause the walnut to become yellow and unacceptable. Coating kumquat with $0.5 \%$ beeswax or $1 \%$ Carnauba wax can also effectively reduce weightlessness during storage.

\section{Summary and prospect}

At present, the preservative technology of fruit and vegetable coating film develops very fast, and the development trend of fruit and vegetable coating film materials is also from having certain toxicity to the direction of low toxicity and even non-toxic and edible. Fresh-keeping coating can improve the utilization rate and quality of fruits and vegetables, so it is an ideal method to store fruits and vegetables. With the widespread application of preservative coating in modern life in recent years, the source, price and performance of preservative coating are paid more and more attention by researchers. The natural polymer has 
the characteristics of wide source, reproducible, degradable, cheap price and good film-forming ability, etc. The modification of natural polymer can prolong the fresh-keeping period of fruits and vegetables and reduce the decay rate of fruits and vegetables. The natural polymer preservative coating has a broad development and application prospect and will play an important role in the field of fruit and vegetable preservation in the future.

This work was supported by Yunnan Province Academician Expert Workstation (202005AF150007), Guangdong Basic and Applied Basic Research Foundation (2019A1515010714), the Natural Science Foundation of Guangdong Province (2018A030313109, 2018A030307015), the Central Public-interest Scientific Institution Basal Research Fund for Chinese Academy of Tropical Agricultural Sciences (1630122017009, 1630122020004), Special Fund for Guangdong Modern Agricultural Industry Technology System Innovation Team Construction (2019KJ140), Guangdong Agricultural Science and Technology Innovation System Management Project (0835190Z22404211C1) and the Science and technology projects of Zhanjiang (2018A02015).

\section{References}

1. R. Siegel, K. Miller, A. Jemal, CA. J. Clin, 66, 22(2016)

2. T.T.Shen, Fresh-keeping and processing .17,03(2017)

3. J. Chen, Yantai Fruit Tree,8,04(2019)

4. Y. M.Zhao, Northern Horticulture, 201,11(2008)

5. W.Zhang,H. Zhao, J. Zhang, Food Chem, 289,160(2019)

6. F.Kittur,N.Saroja,.Eur Food Res Technol ,213,4 (2001)

7. A.Ghaouth,J.Arul,R.Ponnampalam,J Food Sci,56,6(1991)

8. Y.Jiang, L.Yu, Y. Hu, Int J Biol Macromol,139(2019)

9. W.Pasanphan,R.Buettner,S.Chirachancha,Carbohyd res,345,1(2010)

10. S. Torabi, A.R. Mahdavian, Sanei M, Mat Sci Eeng C-Mater, 59,3(2016),

11. U. .Siripatrawan,W.Vitchayakitti, Food Hydrocolloid,695,61(2016)

12. Y.Zhang, M.Zhang, H.Yang, Food Chem, 174,558(2015)

13. Z.Shi , H.Yang, J.Jiao ,Food Chem ,283,92(2019)

14. C.D.Grande-Tovar, C.Chaves-Lopez, A.Serio, Trends Food Sci Tech, 78,61(2018)

15. N.Hasheminejad ,F. Khodaiyan, Food Chem, 309,125(2020)

16. J.Hafsa, M.Smach, Lwt-Food Sci Technol ,68,356(2016)
17. A.Mohammadi, M.Hashemi, S.M.Hosseini,. LwtFood Sci Technol, 65,349(2016)

18. K.Dušan, D.Vladimir, J of Bio Sci,22,2343(2011)

19. B.Sunil, C.Navin, L. Ruchi, Biotechnology and Food Sciences,2,4( 2013)

20. F.Silvia,H.Ana,Eur Food Res Technol, 225,375(2007)

21. B.A. Ashwar, Starch, 67,294(2015)

22. M Maizura, A Fazilah, M .H. Food Chem Toxicol,72,6(2007)

23. A.Taqi, K. A.Askar, L.Mutihac,Food Agr Immunol,24,2(2013)

24. C. H.Chen, W. S. Kuo, L.S. Lai, Food Bioprocess Technol, 6,2(2013)

25. Y. Zhong, Y.F. Li, J Food Sci,77,10(2012)

26. M. Ghasemlou, N. Aliheidaric, R.F.Physical, Carbohyd Polym, 98,1117(2013)

27. P.Carolina, J. Rosa, Food Control,59,737(2016)

28. M.Rohani,Y.Habibi,N.M.Belgacem,G.Ebrahim,A.N .Karimi,A.Dufresne,Eur Polym J,44,8(2008)

29. M.Ljungberg,J.Y.Cavailple,L.Heux.,Polymer,47,18( 2006)

30. M.I.Aranguren,N.E.Marcovich,W.Salgueiro, Polym Test,32,1(2013)

31. A.Alemdar,M.sain,Composites Science and Technology ,68,2(2008)

32. N.Dogan,T.H.McHugh, J Appl PolYm Sci,86,13(2002)

33. L.petersson,I.Kvien,K.Oksman, Compos Sci Technol,67,11(2007)

34. X. Y.Ouyang, Z.Q.Wu, Journal of Hubei University of Technology, 30,5(2015)

35. 35.G .J.Li, Y.X.Zhang, Y.Yang, Guangzhou Chemical Industry, 42,12(2014)

36. X.J.Chen, Journal of Hunan Agricultural University,26,6( 2000)

37. Y. Tong, C.H. Zhang, N.Chang Nan, Agro Food Ind Hi Tec, 11,4(2012)

38. T.M.Martell,M.M.Masson,Carbohyd Polym,198,61(2018)

39. Y.Y.Han,M.YU,L.J.Wang,Food Hydrocolloid ,75,13(2018)

40. T.Wu,S.D.Dai,X.Cong, J Food Process Pres ,41(2017)

41. R.Liu,Y.Liu, Int J Food Sci Tech ,2,9(2017)

42. E.O.Zamudio,G.O.Salcedo,Food Chem, 214,273(2017)

43. M.Hiumarelli,M.D.Hubinger,Food Hydrocolloid,28,59( 2012)

44. G.F.Mehyar,K.A.Ismail,J.Han, J Food Sci,2,77( 2012) 